\title{
Index rerum ad Vol. 149
}

\section{Confecit F. Ammann, Geneve.}

(B) $=$ Buchbesprechungen - Livres nouveaux - Book reviews. $(V)=$ Vortrag - Communication Report.

Alcohol-tobacco optic neuropathy: Analysis of campimetric defects by the static method, 185

Amblyopie; die «Ansteigokklusion» zu $\gamma$ Behandlung der Amblyopie mit exzentrischer Fixation, 456

-; eine Trichterblende bei der Behandlung der Amblyopie mit exzentrischer Fixation, 463

Amblyopiebehandlung; Vergleich der Erfolge der Amblyopiebehandlung mit Euthyskop und Pleopto-phor, 447

Anatomie microscopique de Гæil, v. Histologie

Anterior segment; acquired corneal dystrophy in a case of dysgenesis of the anterior segment of the eye, oligodontia and metacarpal dys-plasia (dysplasia oculodentodigi-talis?), 223 (V)

Arteriitis temporalis, v. Temporal arteritis

Arteriosklerose; über den Wert retinaler Gefäßveränderungen in der Diagnostik der

Arteriosklerose, 248 (B)

Atrophia optica, v. Leber's disease

Augenbinnendruck, v. Glaucoma, congenital, Impressionstonometer, Tonometry

Augengewebe, v. Eye tissues

Augenhintergrundsveränderungen, v. Arteriosklerose, Chloroquine retinopathy, Fundus

diabeticus, Groenblad-Strandberg-Touraine syndrome, Leber's disease, Morbus Boeck,

Netzhaut, Retinal vessels, Retinitis pigmentosa, unilateral, Tapetochoroidal degeneration

Augenkomplikationen, v. Bucarban, Chloroquine retinopathy, Sclero-derma, Temporal arteritis,

Vitamin A deficiency, Uveitis

Augenmuskellähmungen; topogra-phische und aetiologische Analyse von

Augenmuskellähmungen im Elektromyogramm, 359 (V)

Augenmuskeln, v. Augenmuskellähmungen, Muscles oculaires, V-esotropia

Augenverletzungen; allgemeine Be-urteilung von Augenverletzungen, 68

Besnier-Boeck-Schaumann, v. Boeck

Boeck; Fundusveränderungen bei Morbus Boeck, 3

Book reviews, 248 (B), 504-506 (B)

Bucarban; ophthalmologische Er-fahrungen bei Behandlung mit dem oralen Diabetesmittel

Bucarban, 86

Buchbesprechungen, 248 (B), 504-506 (B)

Bulbusabschnitt, vorderer, v. Anterior segment

Buphthalmos, v. Glaucoma, congenital

BZ 55, v. Bucarban

Campimetry, static, v. Optic neuropathy

Cerebral cortex, v. Localization

Champ visuel, v. Optic neuropathy, Perimetrie 
Chemotherapeutica et Antibiotica, v. Bucarban, Jodaugentropfen

508

Index rerum ad Vol. 149

Chloroquine retinopathy; retino-pathy following the use of Chloro-quine and allied substances, $204(\mathrm{~V})$

Choroideremia, v. Tapetochoroidal degeneration

Cibis, Paul A., v. Necrologia

Coefficient de rigidité, v. Tonometry

Collyres iodurés, v. Jodaugentropfen

Colour blindness, v. Dyschromatop-sies

Complications oculaires, v. Bucar-ban, Chloroquine retinopathy, Scleroderma, Temporal

arteritis, Uveitis, Vitamin A deficiency

Congrès, v. Netherlands Ophthal-mological Society, Société Suisse d'Ophtalmologie

Congrès, annonce de, 248, 506

Contact-lens; a new method of applying diagnostic contact-lenses, 227 (V)

Contusion; the mechanics of ocular contusion, 142 (V)

Cornea; megalocornea and mosaic dystrophy of the cornea, 161

Cornea, v. Anterior segment, Dystrophia corneae granulosa, Staphyloma, anterior

Corps vitré, v. Glaskörpercyste

Cours de perfectionnement, v. Société Suisse d'Ophtalmologie

"Crocodile shagreen", v. Mosaic dystrophy

Cystadenoma, papillary; simultaneous occurrence of iridal meta-stasizing papillary cystadenoma and heterolateral palpebral recurring tumour of identical structure, 27

Daltonisme, v. Dyschromatopsies Décollement rétinien, v. Netzhaut-

chirurgie, Retinal detachment Dégénérescence tapéto-choroïdienne,

v. Tapetochoroidal degeneration Dégénérescence tapéto-rétinienne,

v. Retinitis pigmentosa, unilateral Demonstrations, clinical, $146(\mathrm{~V})$,

225 (V) Detachment of retina, v. Netzhaut-

chirurgie, Retinal detachment Diabetes, v. Bucarban

Diagnosis, v. Arteriosklerose, Augen-muskellähmungen, Chloroquine retinopathy, Frühdiagnose,

Fun-dus diabeticus, Ultraschall, Uveitis

Diagnostic; diagnostic des paralysies des muscles oculaires, 344 (V)

Diagnostic contact-lenses; a new method of applying diagnostic contact-lenses, 227 (V)

Diagnostik; Grenzen und Möglich-keiten der ophthalmoneurologi-schen Diagnostik, 312 (V)

Durchleuchtungstest, v. Strabismus

Dyschromatopsies; diagnostic des dyschromatopsies congénitales et acquises, 277 (V)

Dysplasia oculodentodigitalis? Acquired corneal dystrophy in a case of dysgenesis of the anterior segment of the eye, oligodontia and metacarpal dysplasia, 223 (V)

Dystrophia corneae granulosa: Zur individuellen Entwicklung eines Erbleidens, 37

Dystrophie en mosaïque, v. Mosaic dystrophy

Echographie, v. Ultraschall

Electron-microscopy, v. Retino-blastomata

Electro-oculogram, v. Chloroquine retinopathy, Fundus diabeticus, Retinitis pigmentosa,

unilateral

Electroretinogram; a negative component in the b-wave of the human ERG, 230 (V) 
Electroretinogram, v. Chloroquine retinopathy, Fundus diabeticus, Retinitis pigmentosa, unilateral

Electrorétinographie; Гimportance clinique de Гélectrorétinographie, 266 (V)

Elektromyogramm; topographische und aetiologische Analyse von Augenmuskellähmungen im

Elektromyogramm, 359 (V)

EMG, v. Elektromyogramm

EOG, v. Electro-oculogram

ERG, v. Electroret.. ., Electrorét. . .

Experimentelle Forschung, v. Jodaugentropfen

Eye tissues; some mineral constituents of normal human eye tissues ( $\mathrm{Na}-\mathrm{K}-\mathrm{Mg}-\mathrm{Ca}-\mathrm{P}-\mathrm{Cu}), 43$

Index rerum

ad Vol. 149

509

Farbenblindheit, v. Dyschromatop-sies

Farbensinnstörungen, v. Dyschro-matopsies

Fixation, exzentrische, v. Amblyopie

Fluorescein photography of the ocular fundus, 210 (V)

Fortbildung und Orientierung, 68, 152

Fortbildungskurs, v. Schweizerische Ophthalmologische Gesellschaft

Fortschritte der Augenheilkunde (Vol. 14) (Bibliotheca Ophthalmo-logica, Fasc. 63) , 504 (B)

Frühdiagnose; Frühdiagnose und Mikrosymptome, 438 (V)

-;praktische Übungen mit dem Durchleuchtungstest zur Frühdiagnose des Strabismus, 497

Fundus, v. Arteriosklerose, Chloro-quine retinopathy, Groenblad-Strandberg-Touraine

syndrome, Leber's disease, Morbus Boeck, Netzhaut, Betinal vessels, Betinitis pigmentosa,

unilateral, Tapeto-choroidal degeneration

Fundus diabeticus: An evaluation of the pre-retinopathic state, 208 (V)

Further studies and other information, 68, 152

Gefäße, v. Arteriosklerose, Fluorescein photography, Netzhaut, Retinal vessels

Gesichtsfeld, v. Optic neuropathy, Perimetrie

Glaskörpercyste; über einen Fall einer einseitigen Glaskörpercyste: Ein Beitrag zur Kasuistik, 12

Glaucoma, congenital; pathogenesis and treatment of congenital glaucoma, 118 (V)

Goniotomy, v. Glaucoma, congenital

Groenblad-Strandberg-Touraine syndrome, 246 (V)

Heredität, v. Dystrophia corneae granulosa, Keratoconus, Leber's disease, Megalocornea,

Mosaic dystrophy, Osteogenesis imper-fecta, Ptosis, Retinal detachment, Tapetochoroidal degeneration

Histochemistry, v. Eye tissues

Histologie; Handbuch der mikro-skopischen Anatomie des Men-schen. 3. Band, 4. Teil: Das

Auge und seine Hilfsorgane, 505 (B)

Histologie, v. Cystadenoma, papillary, Osteogenesis imperfecta, Retinal prolapse, Retinal

vessels, Retinoblastomata, Staphyloma, anterior, Temporal arteritis, Uveitis

Hornhaut, v. Anterior segment, Cornea, Dystrophia corneae granulosa, Staphyloma, anterior

Impressionstonometer; zur Proble-matik des Im.pressionstonometers von Schiøtz, 96

Industrielle Ophthalmologie, 504 (B)

Inferior oblique muscle; antero-position of the inferior oblique muscle in V-esotropia, 138 (V) 
Instrument, v. Amblyopie, Diagnostic contact-lenses, Impressionstonometer, Kontaktglas,

Strabismus

Intraocular pressure, v. Glaucoma, congenital, Impressionstonometer, Tonometry

Intraokularer Druck, v. Glaucoma, congenital, Impressionstonometer, Tonometry

Iodine containing lotions, v. Jod-augentropfen

Iridocyclochoroiditis, v. Uveitis

Iris, v. Cystadenoma, papillary, Xanthogranuloma, juvenile

Jodaugentropfen; über die Wir-kungsveränderung von Jodaugentropfen im Ablauf der Zeit, 484

Kaninchen, v. Jodaugentropfen Keratoconus, v. Mikrosymptome Kongresse, v. Netherlands

Oph-thalmological Society, Schweizerische Ophthalmologische Gesellschaft Kongreßkalender, 248, 506 Kontaktglas; ein Kontaktglas zur Biomikroskopie der Ora serrata und der Pars plana,

481 Kontaktglas, v. Contact-lens Kontusion, v. Contusion

510

Index rerum

ad Vol. 149

Lapin, v. Jodaugentropfen Leber's disease; a practical aid to

the recognition of Leber's disease,

228 (V) Leber's optic atrophy, v. Leber's

disease Libri, 248 (B), 504-506 (B) Lid, v. Cystadenoma, papillary Livres nouveaux, 248 (B),

504-506 (B) Localization; problems connected

with localization, Ill (V)

Maladie de Leber, v. Leber's disease

Malformations oculaires, v. Anterior segment, Glaskörpercyste, Osteo-genesis imperfecta,

Retinal prolapse, Staphyloma, anterior

Malnutrition, v. Vitamin A deficiency

Médecine psycho-somatique, v. Psychosomatik

Medical meetings, announcement of, 248, 506

Megalocornea; megalocornea and mosaic dystrophy of the cornea, 161

Metastase, v. Cystadenoma, papillary

Methode, v. Amblyopie, Diagnostic contact-lenses, Elektromyogramm, Fluorescein

photography, Kon-taktglas, Ultraschall

Microscopie électronique, v. Retíno-blastomata

Mikroskopische Anatomie des Auges, v. Histologie

Mikrosymptome; Frühdiagnose und Mikrosymptome, 438 (V)

Mineral constituents; some mineral constituents of normal human eye tissues (Na-K-Mg-Ca-P-

$\mathrm{Cu}), 43$

Mißbildungen des Auges, v. Anterior segment, Glaskörpercyste, Osteo-genesis imperfecta,

Retinal prolapse, Staphyloma, anterior

Morbus Boeck; Fundusveränderun-gen bei Morbus Boeck, 3

Mosaic degeneration, v. Mosaic dystrophy

Mosaic dystrophy; megalocornea and mosaic dystrophy of the cornea, 161

Mosaikähnliche Dystrophie, v. Mosaic dystrophy

Muscles oculaires; diagnostic des

paralysies des muscles oculaires,

344 (V) Muskeln, v. Augenmuskellähmun- 
gen, Muscles oculaires, V-eso-

tropia

Necrologia, 80, 248, 506 Netherlands Ophthalmological

Society, 152nd Meeting (1963),

Ill-151 (V); 153rd Meeting (1964),

197-247 (V) Netzhaut; die vaskulären Erkran-

kungen der Netzhaut, 295 (V) Netzhautablösung, v. Netzhaut-

chirurgie, Retinal detachment Netzhautchirurgie; der heutige

Stand der Netzhautchirurgie, 152 Neurochirurgie, v. Tumoren Neuroophthalmologie, v. Ophthalmoneurologische Diagnostik,

Tumoren Névrite optique, v. Optic neuropathy Nordenson, Wilhelm, v. Necrologia Ni-sulfanil$\mathrm{Nj}$-n-butylcarbamid,

v. Bucarban

Ocular tension, v. Glaucoma, congenital, Impressionstonometer, Tonometry

Oculo-dento-digital syndrome, v. Anterior segment

Operation, v. Glaucoma, congenital, Netzhautchirurgie, Retinal detachment, Tumoren, V-

esotropia

Ophtalmologie; Encyclopédie médi-co-chirurgicale (Recueil Na 50, 1963), 504 (B)

Ophtalmologie, Société Suisse d' -, ler Cours de perfectionnement (1964), 250-446 (V)

Ophthalmological Society, Netherlands, 152nd Meeting (1963), Ill-151 (V); 153rd Meeting

(1964), 197-247 (V)

Ophthalmologie, industrielle, 504 (B)

Ophthalmologische Gesellschaft, Schweizerische, 1. Fortbildungs-kurs (1964), 250-446 (V)

Ophthalmoneurologische Diagnostik, Grenzen und Möglichkeiten, 312 (V)

Index rerum ad Vol. 149

511

Optic atrophy, v. Leber's disease Optic neuropathy; alcohol-tobacco optic neuropathy: Analysis of campimetric defects by the static method, 185 Ora serrata, v. Kontaktglas Osteogenesis imperfecta; ocular anomalies in osteogenesis imperfecta, 241 (V)

Paralysies des muscles oculaires,

v. Diagnostic, Elektromyogramm Pars plana, v. Kontaktglas Pathogenesis and treatment of congenital glaucoma, 118 (V) Paupière, v. Cystadenoma, papillary Perfectionnement et orientation,

68, 152 Perimetrie; kurzes Repetitorium der

klinischen Perimetrie, 250 (V) Perimetry, v. Optic neuropathy Physiology, v. Localization

Pleoptik, v. Amblyopiebehandlung Pression oculaire, v. Glaucoma,

congenital, Impressionstonometer,

Tonometry Progrès en ophtalmologie (Vol. 14)

(Bibliotheca Ophthalmologica,

Fasc. 63), 504 (B) Psychosomatik; gibt es eine ophthal-

mologische Psychosomatik?

Brauchen wir eine ophthalmolo-

gische Psychosomatik?, 424 (V) Ptosis, v. Glaskörpercyste

Rabbit, v. Jodaugentropfen

Recherches expérimentales, v. Jodaugentropfen 
Regenbogenhaut, v. Cystadenoma, papillary

Retinal detachment; results of retinal detachment surgery: Operative results in 100 cases, 177

-; hereditary disposition to retinal detachment in two families, 236 (V)

Retinal detachment, v. Netzhaut-chirurgie

Retinal prolapse; congenital anterior staphyloma with retinal prolapse, 21

Retinal vessels; the retinal vessels -comparative ophthalmoscopic and histologic studies on healthy and diseased eyes, 504 (B)

Retinitis pigmentosa, unilateral; does unilateral retinitis pigmentosa really exist? An ERG and EOG study of the fellow eye, 202 (V)

Retinoblastomata; the ultrastructure of retinoblastomata, 53

Retinopathy; retinopathy following the use of Chloroquine and allied substances, 204 (V)

Retinopathy, diabetic, v. Fluorescein photography, Fundus diabeticus

Rieger's dysgenesis, v. Anterior segment

Rigiditätskoeffizient, v. Tonometry

Rigidity coefficient, $v$. Tonometry

Sarkoidose, v. Boeck

Schielen, v. Amblyopie, Augen-muskellähmungen, Muscles oculaires, V-esotropia

Schiøtz-Tonometer, v. Impressions-tonometer

Schweizerische Ophthalmologische Gesellschaft, 1. Fortbildungskurs (1964), 250-446 (V)

Scleral rigidity coefficient, $v$. Tonometry

Scleroderma; generalized sclero-derma with ocular symptoms, 131 (V)

Segment antérieur, v. Anterior segment

Sehschwäche, v. Amblyopie, Amblyopiebehandlung

Selläre und supraselläre Tumoren, Symptomatologie und Chirurgie, 326 (V)

Société Suisse d'Ophtalmologie, ler Cours de perfectionnement (1964), 250-446 (V)

Societies, v. Netherlands Oph-thalmological Society, Schweizerische Ophthalmologische

Gesellschaft, Société Suisse d'Ophtalmologie

Squint, v. Amblyopie, Augenmuskel-lähmungen, Muscles oculaires, V-esotropia

512

Index rerum

ad Vol. 149

Staphyloma, anterior; congenital

anterior staphyloma with retinal

prolapse, 21 Statistische Auswertung, v. Ambly-

opie, Amblyopiebehandlung, Bu-

carban, Netzhautchirurgie, Retinal

detachment Strabismus; praktische Übungen

mit dem Durchleuchtungstest zur

Frühdiagnose des Strabismus, 497 Strabismus, v. Amblyopie, Augen-

muskellähmungen, Muscles

oculaires, V-esotropia Syndrome, v. Anterior segment,

Osteogenesis imperfecta Syndrome of Groenblad-Strandberg-

Touraine, $246(\mathrm{~V})$

Tabak, v. Optic neuropathy Tapetochoroidal degeneration, 221 (V) Temporal arteritis; a fatal case of 
temporal arteritis with ocular symptoms, 121 (V) Tension oculaire, v. Pression

oculaire Therapie, v. Amblyopie, Amblyopiebehandlung, Bucarban, Uvéites Tissus oculaires, v. Eye tissues Tobacco, v. Optic neuropathy Tonometer, v. Impressionstonometer Tonometry; the pressure-volume

relation in tonometry, 81 Trauma, v. Augenverletzungen,

Contusion Tumor, v. Cystadenoma, papillary,

Retinoblastomata, Xantho-

granuloma, juvenile Tumoren; Symptomatologie und

Chirurgie der sellären und supra-

sellären Tumoren, 326 (V) Tumors; ocular and adnexal tumors,

505 (B)

Ultraschall; über die heutigen Auf-fassungen von der Ultraschall-Diagnostik bei intraokularen

Krankheiten, 467

Ultrasons, v. Ultraschall

Ultrasounds, v. Ultraschall

Ultrastructure of retinoblastomata, 53

Unfallophthalmologie, v. Augenverletzungen

Untersuchungstechnik, v. Dys-chromatopsies, Electro-oculo-gram, Electroretinogram, Electrorétinographie, Elektromyogramm, Fluorescein photography, Kon-taktglas, Muscles oculaires, Optic neuropathy, Perimetrie, Strabismus, Ultraschall

Uvéites; traitement sanatorial des uvéites, 416 (V)

Uveitis; Allgemeinleiden und Uveitis, 375 (V)

-; Klinik der Uveitis, Labordiagno-stik, 390 (V)

-; zur pathologischen Anatomie der Uveitis, 405 (V)

Vaisseaux, v. Arteriosklerose, Fluorescein photography, Netz-haut, Retinal vessels

Van der Hoeve's syndrome, v. Osteogenesis imperfecta

Vannas, Mauno, v. Necrologia

Varia, 80, 506

Vererbung, v. Dystrophia corneae granulosa, Keratoconus, Leber's disease, Megalocornea,

Mosaic dystrophy, Osteogenesis imperfecta, Ptosis, Retinal detachment, Tapetochoroidal

degeneration

Verre de contact, v. Contact-lens, Kontaktglas

V-esotropia; anteroposition of the inferior oblique muscle in V-esotropia, 138 (V)

Vessels, v. Arteriosklerose, Fluorescein photography, Netzhaut, Retinal vessels

Visual field, v. Optic neuropathy, Perimetrie

Vitamin A deficiency, epidemiology of, 197 (V)

Vitreous body, v. Glaskörpercyste

$\mathrm{X}$. Xanthogranuloma

juvenile; a case of juvenile xanthogranuloma (naevoxantho-endothelioma) of the iris

151 (V) (published in extenso in: Ophthalmologica

Vol. 147)

Xerophthalmia

v. Vitamin A deficiency 